\title{
Halitosis: new insight into a millennial old problem
}

\author{
Salvatore Corrao
}

Received: 3 January 2011/Accepted: 17 February 2011/Published online: 5 March 2011 (C) SIMI 2011

The problem of halitosis has been reported since ancient times. References have been found in papyrus manuscripts dating back to $1550 \mathrm{BC}$. Hippocrates advised that any girl should have pleasant breath, making sure always to wash her mouth with wine, anise and dill seeds [1].More than 50 years ago, Blackburn [2] investigated halitosis in a caseseries of 73 patients affected by leukemia. He found a peculiar odor of the breath resembling that of a freshly opened corpse. This characteristic smell is not associated with clinical involvement of the gum, mouth, or upper respiratory or alimentary tract. He associated this particular smell with the hematologic disease. Nearly 15 years ago, the role of cadaverine was pointed out as a putative component of halitosis [3]. On the contrary, putrescine, that is similar to cadaverine, is not involved as component of halitosis. Cadaverine, together with putrescine, was first described in 1885 [4] by the German physician Ludwig Brieger (1849-1919). Cadaverine is a toxic diamine usually produced by protein hydrolysis during putrefaction of animal tissue. Cadaverine is also known as 1,5-pentanediamine and pentamethylenediamine, and represents one of the substances possibly implicated in establishing halitosis. Particularly, isolates of Klebsiella and Enterobacter have been found to emit foul odors in vitro that resemble bad breath, with concomitant production of volatile sulfides and cadaverine [5]. When incubated on a sterile denture, enterobacterial isolates produce typical denture foul odor. Moreover, isolates exhibit cell-surface hydrophobic properties when tested for adhesion to acryl and aggregation

\section{S. Corrao $(\square)$}

Dipartimento Biomedico di Medicina Internae Specialistica,

University of Palermo, Piazza delle Cliniche 2,

90147 Palermo, Italy

e-mail: s.corrao@tiscali.it with ammonium sulphate. The results, taken together, suggest that Klebsiella and related Enterobacteriaceae may play a role in denture malodor. However, cadaverine may be produced by other mechanisms as well as oral bacterial growth.

From a conventional point of view, halitosis, fetor oris, oral malodor or bad breath are the general terms used to describe unpleasant breath emitted from a person's mouth regardless of whether the odorous substances in the breath originate from oral or non-oral sources. There are few studies evaluating the prevalence of oral malodor in the general population, with reported rates ranging from 22 to more than $50 \%$. In addition, approximately $50 \%$ of adults and elderly individuals emit socially unacceptable breath, related to physiologic causes, upon arising in the morning [6]. Moreover, there are no universally accepted standard criteria, objective or subjective that define a halitosis patient [7].Thus, this is a clinical problem as common as undervalued by clinicians who have to remember the importance of physical examination as from the mouth's smell for an all-inclusive clinical decision-making. In this issue of Internal and Emergency Medicine, Campisi et al. [8] have carried out an excellent and complete guide for approaching the halitosis symptom. Each of us can learn from this interesting and in-depth review. As the authors state, despite its frequency, this problem is often unaccepted and declared as taboo. However, even if nearly $90 \%$ of patients suffering from halitosis have oral causes, an important percentage of oral malodor cases have an extraoral etiology. They may often be referred to the category of "blood-borne halitosis". According to the Tangerman [9] classification, this is one of the three major categories together with upper respiratory tract and lower respiratory tract causes. Many organ or systemic diseases may be responsible for "blood-borne halitosis" even though some 
food and drugs can also cause this problem. On the other hand, bad breath may be a symptom of infection, inflammation, or malignancy of the upper gastrointestinal or respiratory tracts.

Tongue biofilm seems to be directly involved in the production of oral halitosis, and may have an important role in the success of periodontitis therapy since it is a potential reservoir for periodontal pathogens [10]. It is clear that a successful treatment of halitosis involves an appropriate diagnosis, professional therapy, mechanical plaque control, including tooth brushing and tongue cleaning, possibly combined with the use of an effective antimicrobial mouth-rinse.

Mouth breathing may be a cause of halitosis as well [11]. Consequently, the important issue of causes of mouth breathing has to be considered to correctly set and treat halitosis by tackling underlying etiologies. Moreover, nasal breathing is usually the preferred route of breathing at rest, and mouth breathing occurs when there is nasal obstruction. Thus, subacute rhinosinusitis, allergic rhinitis and other forms of nasal obstructions (including autoimmune diseases such as Wegener's Granulomatosis, a vasculitis syndrome that affects small and medium size vessels) have to be adequately diagnosed and treated to avoid mouth breathing and consequent halitosis. Campisi et al. [8] have also brought in the importance of quantization of halitosis by instrumental evaluation. This is of outstanding importance to exclude psychogenic halitosis. A patient may believe that he or she suffers from halitosis even if it is not the truth. This is the case of pseudo-halitosis that may turn into an obsessive problem known as halitophobia. Some devices exist to support patients with psychogenic halitosis, and the review explains all that very well. From an epistemological point of view, the relevant merit of the Campisi's review has been to have shifted our attention from more and more complex and hyper-technological approaches to patient's problems toward an ancillary symptom whereby clinical reasoning can reconstruct the patient's complexity [12]. The importance of history, physical examination, and any signs and symptoms may be underscored in the age we live in, such as a correct assessment of patient's problems that could simply appear.

In conclusion, halitosis is a complaint with a high social burden, and a high risk of undervaluation as an ancillary symptom. Based on these remarks, a clear algorithm has to be drawn to carry out and optimize an appropriate critical pathway for patients affected by halitosis or psychogenic one. The review of Campisi et al. [8] is a useful tool to approach this issue. Internists and other subspecialists have to rediscover ars medicinae (the art of medicine) to adequately face up to ancillary problems to correctly assess too often undervalued "tip-of-iceberg" conditions such as halitosis. Obviously, ars medicinae does not exclude evidence-based practices, but we all have to remember in our own clinical practice, an aphorism of Sir William Osler, a medicine master of all time, that states "Observe, record, tabulate, communicate. Use your five senses ... Learn to see, learn to hear, learn to feel, learn to smell, and know that by practice alone you can become expert."

Conflict of interest None.

\section{References}

1. Bosy A (1997) Oral malodor: philosophical and practical aspects. J Can Dent Assoc 63(3):196-201

2. Blackburn EK (1957) The breath in acute leukaemia. Br Med J 1(5011):146-147

3. Goldberg S, Kozlovsky A, Gordon D, Gelernter I, Sintov A, Rosenberg M (1994) Cadaverine as a putative component of oral malodor. J Dent Res 73(6):1168-1172

4. Ludwig Brieger (1885) "Weitere Untersuchungen über Ptomaine" [Further investigations into ptomaines]. August Hirschwald, Berlin

5. Goldberg S, Cardash H, Browning H 3rd, Sahly H, Rosenberg M (1997) Isolation of Enterobacteriaceae from the mouth and potential association with malodor. J Dent Res 76(11):1770-1775

6. Rosenberg M (1997) Bad breath: research perspectives. Ramat Aviv: Ramot Publishing-Tel Aviv University Press, Tel Aviv

7. Newman MG (1996) The role of periodontitis in oral malodour: clinical perspectives. In: VanSteenberghe D, Rosenberg M (eds) Bad Breath: a multidisciplinary approach. Leuven University Press, Leuven, pp 3-14

8. Campisi G, Musciotto A, Di Fede O, Di Marco V, Craxì A (2011) Halitosis: could it be more than mere bad breath? Intern Emerg Med. doi:10.1007/s11739-010-0492-4

9. Tangerman A (2002) Halitosis in medicine: a review. Int Dent J 52(Suppl 3):201-206

10. Cortelli JR, Barbosa MD, Westphal MA (2008) Halitosis: a review of associated factors and therapeutic approach. Braz Oral Res 22(Suppl 1):44-54

11. Ng DK, Chow PY, Kwok KL (2005) Halitosis and the nose. Hong Kong Med J 11(1):71-72

12. Nardi R, Scanelli G, Corrao S, Iori I, Mathieu G, Cataldi Amatrian R (2007) Co-morbidity does not reflect complexity in internal medicine patients. Eur J Intern Med 18(5):359-368 